\title{
High-Precision Microwave Spectroscopy of Muonium for Determination of Muonic Magnetic Moment
}

\author{
(MuSEUM Collaboration) \\ (Muonium $\underline{\text { Spectroscopy } \underline{E} x p e r i m e n t} \underline{\text { Using }} \underline{\text { Microwave) }}$ \\ H. A. Torii, Y. Higashi, T. Higuchi, Y. Matsuda, T. Mizutani, M. Tajima, K. S. Tanaka, Y. Ueno \\ (1) Graduate School of Arts and Sciences, University of Tokyo \\ 3-8-1 Komaba, Meguro-ku, Tokyo 153-8902, Japan
}

Y. Fukao, H. Iinuma, Y. Ikedo, R. Kadono, N. Kawamura, A. Koda, K. M. Kojima, T. Mibe, Y. Miyake, K. Nagamine, K. Nishiyama, T. Ogitsu, R. Okubo, N. Saito, K. Sasaki, K. Shimomura, P. Strasser, M. Sugano, A. Toyoda, K. Ueno, A. Yamamoto, M. Yoshida

(2) KEK; 1-1 Oho, Tsukuba, Ibaraki 305-0801, Japan

K. Ishida, M. Iwasaki, O. Kamigaito, D. Tomono

(3) RIKEN; 2-1 Hirosawa, Wako, Saitama 351-0198, Japan

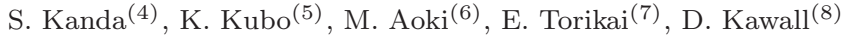 \\ (4) Department of Physics, University of Tokyo; Hongo, Bunkyo-ku, Tokyo 113-0033, Japan \\ (5) International Christian University (ICU); Mitaka, Tokyo 181-8585, Japan \\ (6) Osaka Univeristy; Toyonaka, Osaka 560-0043, Japan \\ (7) University of Yamanashi; Kofu, Yamanashi 400-8511, Japan \\ (8) University of Massachusetts Amherst; MA 01003-9337, USA
}

Published 29 February 2016

\begin{abstract}
The muonium atom is a system suitable for precision measurements for determination of muon's fundamental properties as well as for the test of quantum electrodynamics (QED). A microwave spectroscopy experiment of this exotic atom is being prepared at J-PARC, jointly operated by KEK and JAEA in Japan, aiming at an improved relative precision at a level of $10^{-8}$ in determination of the muonic magnetic moment. A major improvement of statistical uncertainty is expected with the higher muon intensity of the pulsed beam at J-PARC, while reduction of various sources of systematic uncertainties
\end{abstract}

*E-mail of the corresponding arthor (Hiroyuki A. Torii): torii@radphys4.c.u-tokyo.ac.jp.

$\dagger$ Affiliation: also at RIKEN.

$\ddagger$ Current affiliation: Kyoto University; Kyoto 606-8501, Japan.

This is an Open Access article published by World Scientific Publishing Company. It is distributed under the terms of the Creative Commons Attribution 3.0 (CC-BY) License. Further distribution of this work is permitted, provided the original work is properly cited. 
are being studied: those arising from microwave power fluctuations, magnetic field inhomogeneity, muon stopping distribution and atomic collisional shift of resonance frequencies. Experimental strategy and methods are presented in this paper, with an emphasis on our recent development of apparatuses and evaluation of systematic uncertainties.

\section{Introduction}

Muonium is a hydrogen-like exotic atom composed of a positive muon $\left(\mu^{+}\right)$and an electron. It can in fact be regarded as a light "isotope" of hydrogen. The energy level diagram as shown in Fig. 1 is analogous to that of hydrogen, with a difference that the hyperfine splitting is 3 times as large. Purely leptonic muonium is more suited for precision spectroscopy than the hydrogen, where uncertainty of the proton radius prevents accurate comparison between spectroscopy experiments and theoretical calculations. Precision microwave spectroscopy of the ground-state hyperfine states (GS-HFS) of muonium atoms gives a rigorous test of quantum electrodynamics (QED) for bound states as well as precise determination of the magnetic moment $\left(\mu_{\mu}\right)$ of muon and hence its mass $\left(m_{\mu} \propto \mu_{\mu}{ }^{-1}\right)$. The current values of those fundamental constants of muon were determined by the latest experiment at Los Alamos, ${ }^{1}$ already 15 years ago.

Another important outcome expected of HFS spectroscopy is in relation with other experiments dedicated to the measurement of the anomalous magnetic moment $(g-2)_{\mu}$, which is an important physical quantity for the test of the Standard Model and beyond. The magnetic moment to be measured as a ratio to that of the proton $\left(\mu_{\mu} / \mu_{\mathrm{p}}\right)$ is also an indispensable input parameter in deducing the $(g-2)_{\mu}$ value out of their experiments.

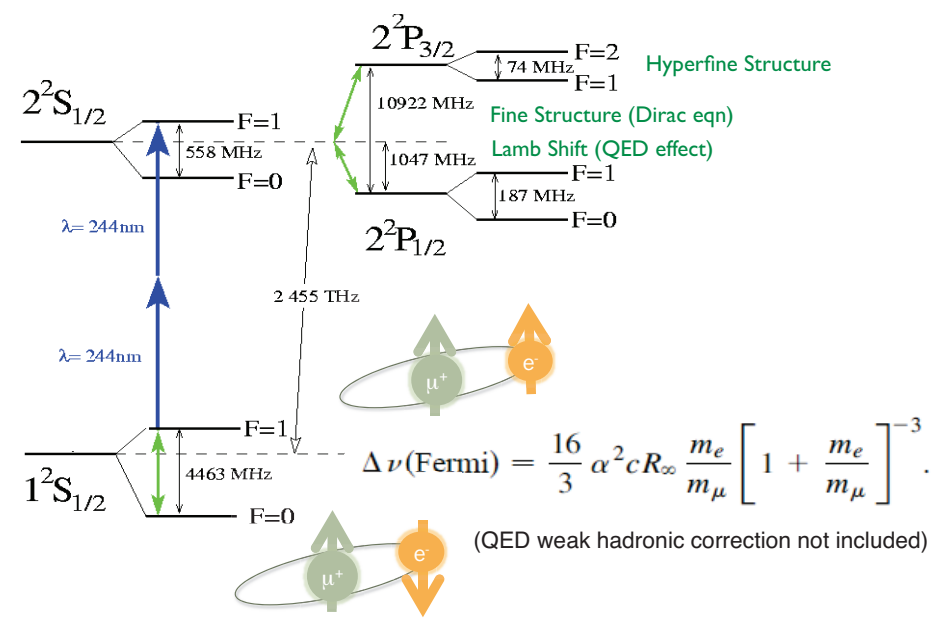

Fig. 1. The energy diagram of the muonium atom (at zero magnetic field). 
Our new experiment planned in coming years aims at a precision at a level of $10^{-8}$ in determination of the muonic magnetic moment, i.e. a factor of several times improvement over the predecessor.

\section{Experimental Strategy}

The ground state hyperfine splitting (HFS) of $\Delta \nu=4.4 \mathrm{GHz}$ is measured with microwave. We do not directly measure this transition at zero magnetic field, but instead, follow the strategy of our predecessor and chose $1.7 \mathrm{~T}$ magnetic field to measure two spin-flip resonance frequencies among 4 magnetic sub-levels. Figure 2 shows the Breit-Rabi diagram of the muonium atom. An important formula holds for the two transitions $\nu_{12}$ and $\nu_{34}$ such that the sum is constant irrespective of the magnetic field and equals the HFS at zero field i.e. $\Delta \nu=\nu_{12}+\nu_{34}$, while the difference $\nu_{12}-\nu_{34}$ is directly related to $\mu_{\mu}$. Thus the sum $\Delta \nu$ gives the QED test while the difference gives the value of the muon magnetic moment.

Figure 3 shows a schematic of the experiment. The muon beams will be stopped in krypton gas to form muonium atoms $(\mathrm{Mu})$ via the electron capture process or the charge exchange reaction $\mu^{+}+\mathrm{Kr} \rightarrow \mathrm{Mu}+\mathrm{Kr}^{+}$. The muon spin is originally $100 \%$ polarized antiparallel to the direction of the muon beam, due to the left-handedness of $\nu_{\mu}$ in the decay of pions: $\pi^{+} \rightarrow \mu^{+}+\nu_{\mu}$. A microwave field will be applied in the RF cavity inside the gas chamber, flipping the muon spin. The resonance manifests itself as directional asymmetry of energetic positrons emitted in favor of the direction of the muon spin when the muon decays, i.e. $\mu^{+} \rightarrow e^{+}+\nu_{e}+\bar{\nu}_{\mu}$.

J-PARC MUSE $H$ line is expected to provide $1 \times 10^{8}$ muons per second in pulses at $25 \mathrm{~Hz}$, and the total number would be $1 \times 10^{15}$ in a running beam time of 100 days, which means 100 times more statistics than the Los Alamos experiment and thus one-order-of-magnitude reduction of statistical uncertainty. Since the statistical uncertainty was the largest component of the total experimental uncertainty in their

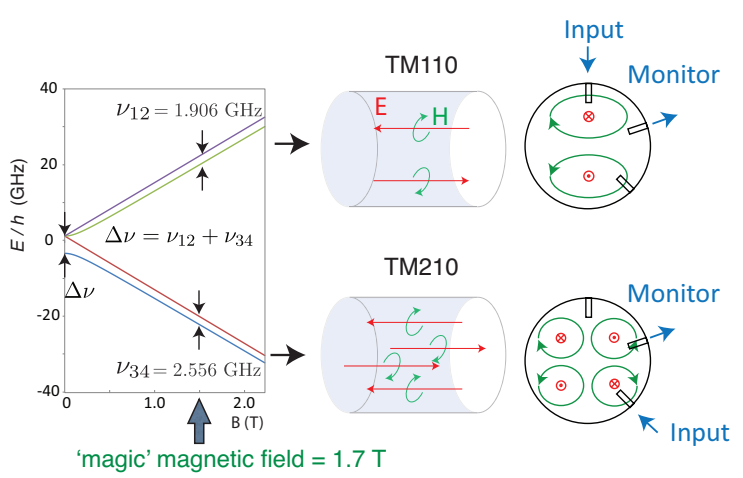

Fig. 2. Left: the Breit-Rabi diagram of the muonium atom shows the energy levels of 4 sublevels as a function of the magnetic field. We chose the 'magic' magnetic field of 1.7 T. Right: TM110 and TM210 modes of the cylindrical cavity were chosen so that the mode frequencies match the resonance frequencies $\nu_{12}$ and $\nu_{34}$, respectively. 


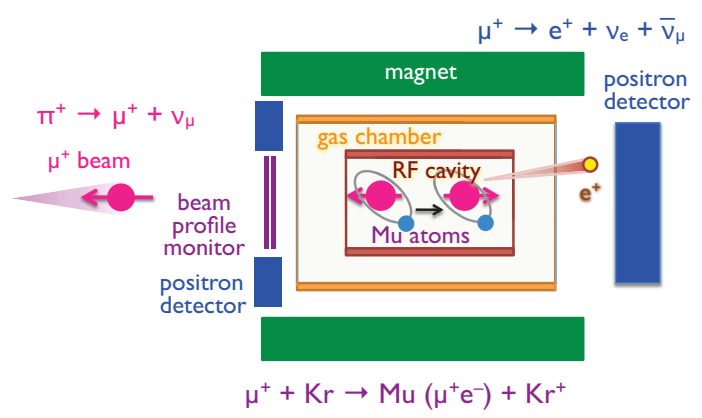

Fig. 3. A schematic of the experiment. Muon beams are stopped in Kr gas to form muonium atoms $\left(\mathrm{Mu}=\mu^{+} e^{-}\right)$in the magnetic field. Resonant muon-spin flip can be detected from asymmetry in the direction of emitted positrons.

experiment, our advantage of higher statistics would surely improve the precision greatly.

\section{Experimental Apparatuses}

We will use TM110 and TM210 microwave modes of a cylindrical cavity, and the magic magnetic field of $1.7 \mathrm{~T}$ was chosen so that the ratio between the two mode frequencies should match the ratio of resonance frequencies $\nu_{12} / \nu_{34}$. The inner diameter of the cavity for the microwave on resonance is $187 \mathrm{~mm}$. We have made a prototype of the cavity out of copper with frequency tuning adjustability by insertion of tuning bars made of alumina. Extensive studies have been made for the resonance frequencies and the $Q$-values of the cavity modes were calculated using microwave simulation codes before fabrication. Our measurements with the prototype was satisfactory, showing a fairly good agreement with the simulation results. ${ }^{2}$

The axial length of the cavity can be determined arbitrarily without affecting the resonance frequencies. We chose $304 \mathrm{~mm}$ length, in comparison to $160 \mathrm{~mm}$ for the Los Alamos experiment. The longer cavity allows more reliable measurements at lower gas pressure. It is generally known in the field of atomic physics that the resonance frequency of atoms and molecules in gas media are subject to collisional shifts due to frequent atomic collision against surrounding atoms. This phenomenon is usually referred to as pressure shift, but the shift is primarily linearly dependent on the collisional frequency which is proportional to the gas density, with a modest dependence on the gas temperature. The shift has in fact been measured for $\mathrm{Mu}$ HFS in past experiments. ${ }^{3}$ Measurements at lower pressures are preferable, in order to minimize the uncertainty in determining the intrinsic resonance frequency by extrapolation to the zero density limit. Los Alamos measurements were performed at around 0.7 and 1.4 atm, while our long cavity will allow measurements at as low as $0.3 \mathrm{~atm}$, still keeping $94 \%$ of the muon stopping distribution inside the cavity.

The windows of the cavity and the gas chamber have to be made thin, in order to allow the muon beam at $4 \mathrm{MeV}$ to penetrate through. Tests have already been 
done for $100 \mu \mathrm{m} \mathrm{Al}$ foil and $25 \mu \mathrm{m} \mathrm{Cu}$ foils with $100 \mathrm{~mm}$ diameter. The muon position will always be monitored by a set of beam profile monitors to be inserted upstream of the chamber in the beam line. We need two layers ( $x$ and $y$ ) for a two-dimensional image of the muon beam profile. The thickness of each layer has to be constrained to not more than $100-200 \mu \mathrm{m}$ for the same reason above. Our first idea of using scintillator strips turned out difficult if uniformity of such a small thickness is required. We are now developing a beam profile monitor made up of $100 \mu$ m-diameter scintillation fibers assuring good light transmission, arrayed and glued together to form a $150 \mu \mathrm{m}$-thick sheet.

The gas temperature needs to be controlled to a sub-kelvin level and the pressure to be monitored accurately. Gas purity is required to a ppm level so as to avoid chemical quenching of muonium polarization and possible collisional shift of the resonance frequency by highly reactive gases such as $\mathrm{H}_{2}$ or $\mathrm{O}_{2}$.

Choice of a longer cavity imposes severer requirement on the magnet. Field homogeneity of less than 1 ppm with absolute calibration is required in a large spheroidal region of $200 \mathrm{~mm}$ diameter $\times 300 \mathrm{~mm}$ length. The field strength are measured by water NMR probes (so that the magnetic moment $\mu_{\mu}$ is measured in reference to that of proton $\mu_{\mathrm{p}}$ ) at some 500 different positions to obtain a field map. The field will be corrected by shim coils and by iron shims iteratively inserted until the homogeneity is satisfactory. The positions and the numbers of iron shims to be inserted on the wall of the bore of the magnet will be calculated by solving the matrix equations with the Single Value Division Method. We also need to take care of possible shift of the NMR frequency due to a so-called shape effect arising from the non-spherical shape of the water volume of the NMR probe.

The muon spin at its decay is detected by measuring the directional asymmetry of the emitted positron. In order to cope with the high event rate characteristic of the pulsed beam, highly segmented scintillators and multi-pixel photon counters (MPPCs) will be used, and the signals are processed by ASIC-based ASD and FPGA multi-hit TDC. A prototype has been developed and beam tests have been performed. ${ }^{4}$

\section{Estimation of Uncertainty}

It is really a challenge to determine the centroid of the $100-\mathrm{kHz}$-wide resonance line to a level of a hertz. Selection of long-lived muonium (the so-called 'old muonium' method) gives narrower resonances (with side peaks) because of the principle that longer observation time gives less uncertainty in energy, at the sacrifice of statistics. Great improvement in the muon number at J-PARC is expected to reduce the major component of the current uncertainty limit, but several other systematic uncertainties need to be verified when a precision of $10^{-8}$ for $\mu_{\mu}$ is pursued.

The microwave power has a direct impact on the resonance intensity. Naïvely speaking, $1 \%$ change of the power would result in $1 \%$ change of the resonance signal (unless the resonance is saturated). Any power fluctuation or drift during the 


\section{H. A. Torii et al.}

resonance scan would immediately deteriorate the resolution of the resonance frequency. Also, the position distribution of muonium atoms is of importance because the microwave field strength is different from position to position.

So we performed a series of simulations where the 3D maps of muon distribution, microwave field and detection efficiency were combined. Resonant transition probability was calculated at each position of the muonium atom by numerically solving the time evolution of Bloch equation to obtain a simulated resonance signal for various situations. Whether the muon has flipped its spin or not was determined by throwing a 'dice' with the calculated probability. The position was randomly chosen with the Monte Carlo method, and in accordance with the muon stopping distribution. By summing up all muon positions, the overall simulated resonance data can be plotted. This data set was then fitted with a theoretical line, in the same manner as the real experimental data will be fitted. The resultant values of the fit parameters gave an estimation of the shift and the uncertainty in determination of the resonance frequency.

As an example of experimental situations, suppose the power was gradually changed by $0.1 \%$ along with the frequency being scanned over the resonance region of $500 \mathrm{kHz}$, then the resultant centroid deviated from the original value by $49 \mathrm{~Hz}$. The narrower 'old muonium' spectrum turned out more robust and the shift was $5.5 \mathrm{~Hz}$. Another example checked centroid deviation caused by the drift of muon position distribution. We verified that stationary displacement of the distribution does not cause any frequency shift whilst position drift by a few centimeters during the frequency scan does have a sizable effect. This is the reason why we need constant monitoring of the muon beam profile. All these types of realistic simulations help indispensable understanding of the systematic uncertainties in determination of the resonance frequencies.

\section{Summary}

In summary, we the MuSEUM collaboration are aiming at precision spectroscopy of the ground-state hyperfine splitting of the muonium atom. We are making steady progress in development of various parts of our experimental apparatuses which are all designed to reduce systematic uncertainties being studied.

\section{References}

1. W. Liu et al., Phys. Rev. Lett. 82 (1999) 711.

2. K. S. Tanaka et al., JPS Conf. Proc. 2 (2014) 010405.

3. D. E. Casperson et al., Phys. Lett. B 59 (1975) 397 ; V. W. Hughes et al., Phys. Rev. Lett. 87 (2001) 111804.

4. S. Kanda on behalf of MuSEUM Collaboration, Proceeding of the 2nd International Symposium on Science at J-PARC (J-PARC 2014), JPS Conference Proceedings, to be puslished (2015). 